\title{
Erratum
}

\section{Dehydroascorbate reductase and glutathione reductase play an important role in scavenging hydrogen peroxide during natural and artificial dehydration of Jatropha curcas seeds}

Samar A. Omar, Nabil I. Elsheery, Hazem M. Kalaji, Zeng-Fu Xu, Song-Quan Song, Robert Carperntier, Choon-Hwan Lee, and Suleyman I. Allakverdiev

Volume 55, page 469, there is an error in the corresponding authorship. Corresponding authors are Zeng-Fu Xu and SongQuan Song. E-mail: zfxu@xtbg.ac.cn; sqsong@ibcas.ac.cn

Page 478, in Acknowledgements, "This work was supported by the Knowledge Innovation Program of the Chinese Academy of Science (to SAO)." should be change to "This work was supported by the Knowledge Innovation Program of the Chinese Academy of Sciences (grant no. KSCX2-YW-Z-0723) and the Top Science and Technology Talents Scheme of Yunnan Province (grant No. 2009CI123) to ZFX." 